\title{
The European gonococcal antimicrobial surveillance programme, 2009
}

M J Cole (michelle.cole@hpa.org.uk) ${ }^{1}$, M Unemo $^{2}$, S Hoffmann ${ }^{3}$, S A Chisholm ${ }^{1}$, C A Ison ${ }^{1}$, M J van de Laar ${ }^{4}$

1. Health Protection Agency, London, United Kingdom

2. Örebro University Hospital, Örebro, Sweden

3. Statens Serum Institut, Copenhagen, Denmark

4. European Centre for Disease Prevention and Control, Stockholm, Sweden

Citation style for this article:

Cole MJ, Unemo M, Hoffmann S, Chisholm SA, Ison CA, van de Laar MJ. The European gonococcal antimicrobial surveillance programme, 2009.

Euro Surveill. 2011;16(42):pii=19995. Available online: http://www.eurosurveillance.org/ViewArticle.aspx?Articleld=19995

Article published on 20 October 2011

Neisseria gonorrhoeae antimicrobial susceptibility is monitored in the European Union (EU) and the European Economic Area (EEA) by the European gonococcal antimicrobial surveillance programme (EuroGASP). Results from 17 EU/EEA Member States in 2009 showed that $5 \%$ of isolates had decreased susceptibility to cefixime, an upward trend in the minimum inhibitory concentrations of ceftriaxone and a high prevalence of resistance to ciprofloxacin (63\%) and azithromycin (13\%). These results are of public health value and highlight the need for healthcare professionals to be aware of possible cefixime treatment failures. Euro-GASP is being implemented in additional EU/EEA Member States to achieve greater representativeness. In addition, Euro-GASP aims to set up a system which will allow biannual reporting of antimicrobial resistance in the EU/EEA, with a transition from centralised towards decentralised testing, and will link epidemiological data to laboratory data to enhance surveillance. The benefits of this approach include more timely detection of emerging trends in gonococcal resistance across the EU/EEA and the provision of a robust evidence base for informing national and European guidelines for therapy.

\section{Introduction}

Surveillance systems to detect the emergence and spread of Neisseria gonorrhoeae antimicrobial resistance (AMR) are essential to ensure patients receive the most appropriate therapy for gonorrhoea treatment. Over the years, the gonococcus has demonstrated the ability to develop resistance to antimicrobial drugs used for therapy $[1,2]$, and the subsequent worldwide spread of resistant strains [3-8] has shown that the antimicrobial susceptibility of this bacterium needs to be monitored closely to inform public health control. The current European recommended therapy for gonorrhoea [9] consists of the extended-spectrum cephalosporins (ESCs) ceftriaxone and cefixime, which are also used in most other countries worldwide [1]. Decreased susceptibility to ESCs was first recognised in 2001 in Japan [10] and subsequent reports of worldwide spread $[1,11,12]$, including reports from Europe
$[6,13]$ are becoming more common. The loss of ESCs for the treatment of gonorrhoea would be a major public health concern. The danger that gonorrhoea may become untreatable is real, particularly in light of treatment failure of cefixime first documented in Japan [14] and more recently in Norway and the United Kingdom $[15,16]$ as well as the decreasing susceptibility to ceftriaxone globally $[1,2]$.

Six countries within the European Union and the European Economic Area (EU/EEA) perform national surveillance of $N$. gonorrhoeae $A M R$ and regularly publish their results [6,17-21]. A European approach is however useful to obtain valid and comparable data, to encompass countries without a surveillance system, and to monitor the movement of resistant strains throughout Europe. The European gonococcal antimicrobial surveillance programme (Euro-GASP) was established in 2004 [22] and continued until 2008 [7] as part of the European Commission-funded European surveillance of sexually transmitted infections (ESSTI) project. Their results from 2004 to 2008 [7] revealed a high mean prevalence of ciprofloxacin resistance $(>30 \%)$ across the participating countries, a varying mean annual prevalence of azithromycin resistance (2-8\%), along with high-level azithromycin resistance ( $256 \mathrm{mg} / \mathrm{L}$ ) in Scotland and Ireland. Of utmost concern was the upward trend in the minimum inhibitory concentration (MIC) of ceftriaxone [7]. These results, along with the lack of alternative treatment options, highlight the need for a pan-European and international approach monitoring the emergence and spread of gonococcal resistance to ensure the most appropriate therapies are administered and preserved.

In 2009, the European Centre for Disease Prevention and Control (ECDC) took over the responsibility of coordinating the enhanced surveillance of sexually transmitted infections (STI) in the EU/EEA. The European STI surveillance expert network was established, consisting of nominated experts in epidemiology and microbiology. The first European STI surveillance report on the period from 1990 to 2009 has been published recently 
and showed that the number of gonorrhoea cases per 100,000 population exceeded five per 100,000 population in many European countries [23], a sign that gonorrhoea remains a public health problem also in the EU/ EEA. The objectives of the enhanced STI surveillance in the EU/EEA include strengthening the laboratory diagnostics for bacterial STIs, and ECDC has launched and funds an STI microbiology project which focuses on the surveillance of gonococcal antimicrobial susceptibility (Euro-GASP). Its main purpose is to agree on a new protocol for a European gonococcal antimicrobial surveillance programme, including an external quality assessment (EQA) scheme, and to provide training in STI laboratory diagnostics. Euro-GASP, aims to provide susceptibility data for a range of therapeutically relevant antimicrobial drugs in a timely manner by reporting trends and developments in gonococcal antimicrobial resistance in the EU/EEA and by linking susceptibility data with epidemiological information.

This article summarises the first gonococcal susceptibility data generated from Euro-GASP in 2009, as published by ECDC [24].

\section{Material and methods}

Members of the European STI surveillance network were invited to participate in Euro-GASP during 2009. Seventeen EU/EEA Member States (Austria, Belgium, Denmark, France, Germany, Greece, Italy, Latvia, Malta, the Netherlands, Norway, Portugal, Slovakia, Slovenia, Spain, Sweden and the United Kingdom) agreed to participate and to collect isolates for susceptibility testing from a number of laboratories. Two participating countries collected specimens from laboratories with a comprehensive coverage of all diagnosed gonorrhoea in their country (Denmark and Sweden). Eight laboratories had good national coverage, defined as representations from all areas of the country but not every case of diagnosed gonorrhoea (Belgium, France, Greece, Latvia, Malta, Norway, Portugal and Spain). Seven laboratories had good coverage of a particular region of the country (Austria, Germany, Italy, the Netherlands, Slovakia, Slovenia and the United Kingdom).

Each country was asked to contribute 110 consecutive isolates of $N$. gonorrhoeae with the aim of retrieving 100 isolates from each country. Isolates were collected from 1 October 2009 until 110 isolates were collected, ending latest on 31 December 2009. Some countries (Austria, Germany, Greece, Italy, Latvia, Malta, Norway, Portugal and Slovenia) started collecting isolates before the start date, to compensate for a low collection rate. Isolates from the United Kingdom were collected during the collection period of the national gonococcal resistance to antimicrobials surveillance programme (GRASP) [6] in June and July 2009 as national isolates were not available outside of the GRASP collection period. Laboratories were requested to collect only one isolate per patient from those who were infected at multiple sites or at multiple times within a four week period; rectal followed by urethral specimens were preferred from males and cervical specimens from females. Pure cultures of N. gonorrhoeae were sent frozen on dry ice to one of three laboratories for susceptibility testing (Health Protection Agency (HPA), London, UK; Statens Serum Institut, Copenhagen, Denmark and Örebro University Hospital, Örebro, Sweden). Isolates were recovered from the frozen stock and confirmed to be $N$. gonorrhoeae by Gram staining, oxidase test, and Microtrak test (Trinity Biotech, Wicklow, Ireland).

The antibiotic susceptibility of the isolates was assessed using the agar dilution breakpoint technique for ciprofloxacin, azithromycin, and spectinomycin as previously described [7]. Susceptibility to gentamicin was determined by the full agar dilution technique as previously described [25], and isolates exhibiting an MIC to gentamicin of $>8 \mathrm{mg} / \mathrm{L}$ were additionally tested by Etest ( $A B$ bioMérieux, Solna, Sweden). Furthermore, Etests were used for MIC determination of ceftriaxone and cefixime. Etests were also performed for azithromycin, i.e. if isolates displayed azithromycin resistance by the agar dilution breakpoint technique. World Health Organization (WHO) quality assurance and control strains ( $A, G, J, K, M, O$ and $P$ ) specifically chosen for gonococcal susceptibility testing were used for quality control [26]. All isolates were tested for penicillinase production using the chromogenic reagent Nitrocefin (Oxoid, Basingstoke, UK). Breakpoints, which are the MICs defined for determining category of resistance, are shown in Table 1.

\section{Results}

Over the 2009 collection period, 1,471 isolates were collected and 1,367 isolates were successfully recovered from the frozen cultures and confirmed to be $N$. gonorrhoeae (93\% retrieval rate). A total of 1,366 isolates were included in the analysis after the removal of one duplicate isolate. The number of isolates tested from

\section{TABLE 1}

Neisseria gonorrhoeae breakpoints used to determine category of resistance, Euro-GASP, 2009

\begin{tabular}{|l|c|c|c|}
\hline \multirow{2}{*}{ Antimicrobial } & \multicolumn{3}{|c|}{ MIC breakpoint (mg/L) } \\
\cline { 2 - 4 } & $\mathrm{R} \geq$ & $\mathrm{I}$ & $\mathrm{S} \leq$ \\
\hline Azithromycin & 1 & - & 0.5 \\
\hline Cefixime & TBD & & $0.125^{\mathrm{a}}$ \\
\hline Ceftriaxone & TBD & & $0.125^{\mathrm{a}}$ \\
\hline Ciprofloxacin & 1 & $0.12-0.5$ & 0.06 \\
\hline Gentamicin & \multicolumn{3}{|c|}{ TBD } \\
\hline Spectinomycin & 128 & \multicolumn{3}{|c|}{} \\
\hline
\end{tabular}

I: intermediate; MIC: minimum inhibitory concentration; $\mathrm{R}$ : resistance; S: susceptible; TBD: to be determined.

European Committee on Antimicrobial Susceptibility Testing (EUCAST) [27] breakpoints were used, except for ciprofloxacin, and azithromycin intermediate resistance where breakpoints used in GRASP were adhered to [6].

a Strains with MIC $>0.125 \mathrm{mg} / \mathrm{L}$ were defined as having decreased susceptibility/resistance (appropriate laboratory and clinical correlates are lacking for evidence-based determination for this breakpoint). 
each country varied from nine (Latvia) to 120 (United Kingdom) (Table 2).

Data from all countries were combined to determine the overall resistance rates to azithromycin, ciprofloxacin and penicillin (plasmid mediated high-level resistance) in 2009 (Table 2). Overall 63\% of all isolates displayed resistance to ciprofloxacin, with a prevalence of ciprofloxacin resistance of more than $5 \%$ in any given country (Table 2). Azithromycin resistance was shown to be $13 \%$, with the most frequently occurring MIC of azithromycin-resistant isolates being close to the breakpoint of $1.0 \mathrm{mg} / \mathrm{L}$. None of the isolates displayed high-level resistance to azithromycin (>256 mg/L). Highlevel resistance to penicillin (penicillinase-producing N. gonorrhoeae) was seen in $12 \%$ of isolates, and no resistance to spectinomycin $(>64 \mathrm{mg} / \mathrm{L})$ was detected in 2009.

Five per cent $(70 / 1,366)$ of the isolates displayed decreased susceptibility (>0.125 mg/L) to cefixime (Table 3, Figure 1). All 70 isolates additionally harboured resistance to ciprofloxacin. The decreased susceptibility to cefixime was detected in 10 countries, in five of which more than $5 \%$ of isolates had decreased susceptibility to cefixime (Table 3 ).
No decreased susceptibility to ceftriaxone (>0.125 $\mathrm{mg} / \mathrm{L}$ ) was detected in 2009 and the most frequently occurring (modal) MIC to ceftriaxone in the isolates was $0.008 \mathrm{mg} / \mathrm{L}$ (Figure 2).

The MIC distribution of gentamicin in European isolates from 2009 has previously been described; both the $\mathrm{MIC}_{50}$ and $\mathrm{MIC}_{90}$ of gentamicin was $8 \mathrm{mg} / \mathrm{L}$ with an MIC range of $1-16 \mathrm{mg} / \mathrm{L} \mathrm{[25].}$

\section{Discussion}

Five per cent of all $N$. gonorrhoeae isolates collected in the 17 countries had decreased susceptibility to cefixime. Cefixime was included in the list of antimicrobial drugs monitored by Euro-GASP for the first time in 2009 , so it is not known if this proportion of $5 \%$ is an increase from previous years. Even though the majority of isolates ( $58 \%$ ) showed low MICs $(\leq 0.016 \mathrm{mg} / \mathrm{L})$, it is of concern that isolates displaying higher MICs (>0.125 $\mathrm{mg} / \mathrm{L}$ ) exist. Even though the relationship between the MIC for cefixime and treatment failure remains poorly understood and appropriate resistance breakpoints are yet to be described, treatment failure could become an increasing problem in Europe if MICs continue to increase. Therefore all European countries that use cefixime for treatment should alert appropriate health professionals to monitor for treatment failure. Advisory

TABLE 2

Neisseria gonorrhoea resistance to azithromycin, ciprofloxacin, and high-level resistance to penicillin, and strains fully susceptible to all antimicrobial drugs, 17 EU/EEA countries, $2009(n=1,366)$

\begin{tabular}{|c|c|c|c|c|c|}
\hline \multirow[b]{2}{*}{ Country } & \multirow[b]{2}{*}{$\begin{array}{c}\text { Number of isolates } \\
\text { tested }^{\mathrm{a}}\end{array}$} & \multicolumn{3}{|c|}{ Resistance } & \multirow[b]{2}{*}{ Fully susceptible } \\
\hline & & $\begin{array}{c}\text { Azithromycin } \\
\mathrm{n}(\%)\end{array}$ & $\begin{array}{c}\text { Ciprofloxacin } \\
n(\%)\end{array}$ & $\begin{array}{l}\text { PPNG } \\
\mathrm{n}(\%)\end{array}$ & \\
\hline Austria & 104 & $30(29)$ & $83(80)$ & $9(9)$ & $21(20)$ \\
\hline Belgium & 110 & 16 (15) & $74(67)$ & $24(22)$ & $32(29)$ \\
\hline Denmark & 119 & $55(46)$ & $83(70)$ & $7(6)$ & $30(25)$ \\
\hline France & 104 & $19(18)$ & $45(43)$ & $6(6)$ & $53(51)$ \\
\hline Germany & 45 & 0 & $33(73)$ & $3(7)$ & $12(27)$ \\
\hline Greece & 110 & $9(8)$ & $74(67)$ & $4(4)$ & $32(29)$ \\
\hline Italy & 70 & $20(29)$ & $53(76)$ & $5(7)$ & $16(23)$ \\
\hline Latvia & 9 & 0 & $1(11)$ & 0 & $8(89)$ \\
\hline Malta & 22 & $1(5)$ & $20(91)$ & 0 & 0 \\
\hline The Netherlands & 114 & $3(3)$ & $56(49)$ & $5(4)$ & $58(51)$ \\
\hline Norway & 110 & $2(2)$ & $88(80)$ & $41(37)$ & 14 (13) \\
\hline Portugal & 79 & 0 & $27(34)$ & $13(16)$ & $46(58)$ \\
\hline Slovakia & 15 & $1(7)$ & $15(100)$ & $1(7)$ & 0 \\
\hline Slovenia & 24 & $2(8)$ & $19(79)$ & $3(13)$ & $3(13)$ \\
\hline Spain & 103 & $6(6)$ & $67(65)$ & $7(7)$ & $33(32)$ \\
\hline Sweden & 108 & $11(10)$ & $77(71)$ & 37 (34) & $25(23)$ \\
\hline United Kingdom ${ }^{b}$ & 120 & $5(4)$ & $42(35)$ & $7(6)$ & $76(63)$ \\
\hline Total & 1,366 & $180(13)$ & 857 (63) & 172 (13) & 459 (34) \\
\hline $95 \% \mathrm{Cl}$ & & $(11.4-15)$ & $(60.2-65.3)$ & $(10.8-14.4)$ & $(31.1-36.1)$ \\
\hline
\end{tabular}

$\mathrm{Cl}$, confidence interval of the total \% mean; EU/EEA: European Union and European Economic Area; PPNG: penicillinase-producing Neisseria gonorrhoeae.

a Number of isolates tested varied due to differences in test methodology, laboratory structure within each country and total number of reported gonorrhoea cases [23]

b 2009 isolates from the United Kingdom were only from England and Wales. 
groups who recommend treatment options, should consider higher doses or alternative regimes [28]. The loss of cefixime as an oral treatment option for gonorrhoea across the EU/EEA may have severe implications because the use of the parenterally administered ceftriaxone is more expensive and there are currently no new alternative treatments.

Ceftriaxone continues to be an appropriate treatment for gonorrhoea in the EU/EEA as all tested isolates were susceptible. However the modal MIC of isolates to ceftriaxone has increased to $0.008 \mathrm{mg} / \mathrm{L}$ in 2009

\section{TABLE 3}

Prevalence of decreased susceptibility to cefixime among Neisseria gonorrhoea isolates from 10 EU/EEA countries, $2009(\mathrm{n}=908)$

\begin{tabular}{|l|c|}
\hline $\begin{array}{l}\text { Country } \\
\text { (total number of isolates tested) }\end{array}$ & $\begin{array}{c}\text { Isolates with DS-cefixime } \\
\text { Number (\%) }\end{array}$ \\
\hline Austria (104) & $22(21.2)$ \\
\hline Italy (70) & $13(18.6)$ \\
\hline Denmark (119) & $18(15.1)$ \\
\hline Slovenia (24) & $2(8.3)$ \\
\hline Belgium (110) & $7(6.4)$ \\
\hline Sweden (108) & $3(2.8)$ \\
\hline Germany (45) & $1(2.2)$ \\
\hline France (104) & $2(1.9)$ \\
\hline The Netherlands (114) & $1(0.9)$ \\
\hline Norway (110) & $1(0.9)$ \\
\hline
\end{tabular}

DS: decreased susceptibility; EU/EEA: European Union and European Economic Area.

Overall level of decreased susceptibility to cefixime in the 17 countries participating in Euro-GASP: $5.1 \%$ (70/1,366). Seven countries are not included in the table as no isolates displaying decreased susceptibility to cefixime were detected.

\section{FIGURE 1}

Distribution of minimum inhibitory concentrations of Neisseria gonorrhoea isolates for cefixime, 17 EU/EEA countries, $2009(n=1,366)$

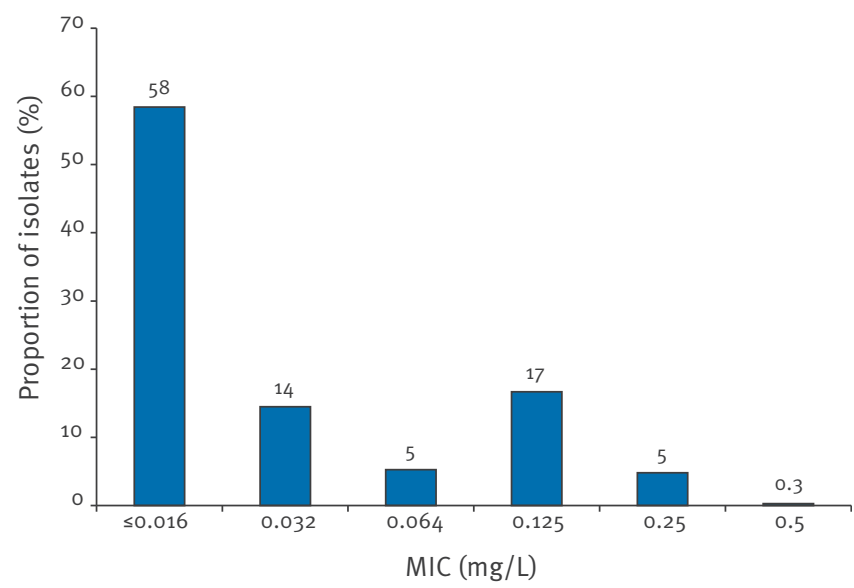

MIC: minimum inhibitory concentration.

Etest MIC values inbetween two-fold dilutions have been rounded up to next two-fold dilution. compared with $\leq 0.002 \mathrm{mg} / \mathrm{L}$ in 2004 [22]. In addition, the proportion of isolates that display MICs of $\geq 0.094$ $\mathrm{mg} / \mathrm{L}$ for ceftriaxone has been increasing annually (2.9\% in 2009). This upward trend and recent identification of ceftriaxone treatment failure for pharyngeal infection $[29,30]$ make it clear that emerging resistance to ceftriaxone needs to be carefully monitored.

There is no overall trend in the azithromycin resistance of $N$. gonorrhoeae isolates: the level of azithromycin resistance increased from 2008 to 2009 ( $2 \%$ to $13 \%$ ), but had decreased from 2007 to 2008 ( $7 \%$ to $2 \%$ ) [7]. This is most probably due to the fact that the modal MIC of resistant isolates falls on a breakpoint, as previously described [7]. Azithromycin is not a recommended treatment for gonorrhoea in Europe, however it is important to continue monitoring for resistance as azithromycin may be a therapeutic option in particular situations or in combination therapy [31]. The high prevalence of ciprofloxacin resistance, previously reported by ESSTI [7,22], continued and further increased by $12 \%(51 \%$ to $63 \%)$ in 2009 in spite of changes to the European treatment guidelines [9] that recommend that ciprofloxacin should not be used as first line therapy, and that, together with azithromycin, it should not be used for empirical treatment, unless isolates are known to be susceptible or local resistance rates are known to be less than $5 \%$.

The ECDC in close collaboration with the STI expert network has established Euro-GASP as a programme for sentinel surveillance of $N$. gonorrhoeae AMR across the EU/EEA. The programme aims to provide longitudinal robust data to inform treatment guidelines at this crucial time when gonorrhoea is becoming difficult to treat. Describing the prevalence of resistance or decreased

\section{FIGURE 2}

Distribution of minimum inhibitory concentrations of Neisseria gonorrhoea isolates for ceftriaxone, 17 EU/EEA countries, $2009(\mathrm{n}=1,366)$

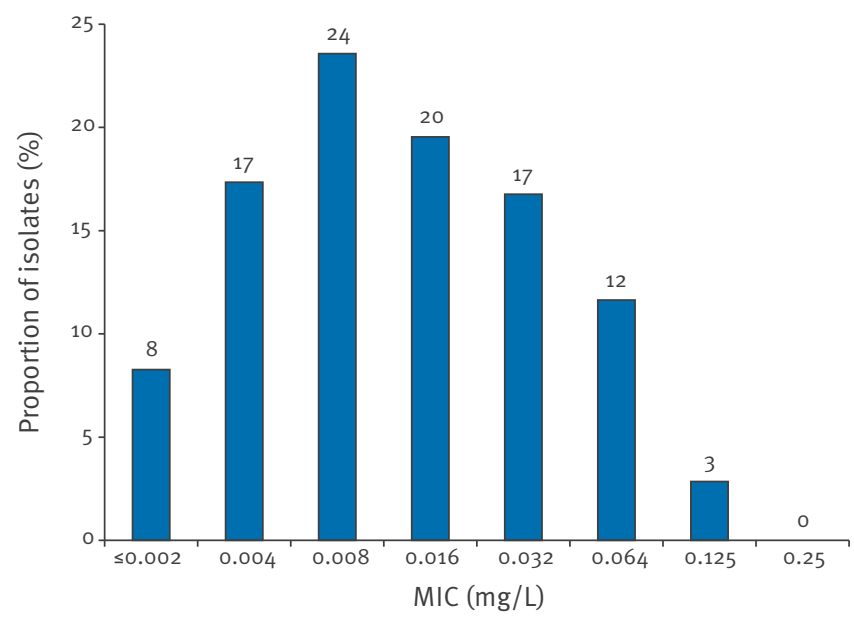

MIC: minimum inhibitory concentration.

Etest MIC values inbetween two-fold dilutions have been rounded up to next two-fold dilution. 
susceptibility across Europe by combining countries gives the study more power due to the larger sample size ( $n>1,000)$. However the low number of isolates collected from some countries limits the conclusions that can be drawn for individual countries. Increasing the number of isolates to obtain a representative sample is a priority for Euro-GASP. The reasons for low collection numbers vary from a lack of resources, and differences in clinical and diagnostic practices such as molecular testing, to a low number of gonorrhoea cases. Ultimately, the success of Euro-GASP depends upon the availability of $N$. gonorrhoeae cultures, and it is extremely important that the countries' capacity for collection of $N$. gonorrhoeae cultures is strengthened to obtain adequate numbers of isolates. Coverage will be extended to sample gonococcal isolates from as many EU/EEA countries as possible and a system of combined centralised and decentralised testing will be implemented to allow biannual reporting of AMR and improve timeliness of trend data. Furthermore, the surveillance of gonococcal AMR will be enhanced by linking laboratory data to appropriate epidemiological data. This will facilitate the future improvement of sample representativeness by providing information on the patients sampled in individual countries and the better understanding of risk factors associated with emerging resistance patterns. Euro-GASP will support countries in producing sustainable gonococcal resistance surveillance programmes by providing EQA and training. As resistance continues to emerge, the panel of antimicrobial agents tested will be constantly reviewed and may need to include new or old drugs. For example, gentamicin, a potential future treatment option (for use in single therapy or especially in combination therapy), has been included in Euro-GASP since 2009. An evaluation of the current programme is foreseen in 2011. The modernisation and development of Euro-GASP has shown how a surveillance system can remain agile to keep up with the ever changing, versatile gonococcus.

\section{Acknowledgments}

We acknowledge the European STI surveillance network for their contribution in the development and implementation of Euro-GASP and the submission of gonococcal isolates and data. We further wish to thank Dr Jørgen Skov Jensen and Prof. Hans Fredlund for support and advice. Nerteley Quaye, Lene Berthelsen, Ronza Hadad and Emma Johansson for performing the laboratory work.

\section{Funding}

The study was funded by the European Centre for Disease Prevention and Control, contract number ECD.2312.

\section{References}

1. Lewis DA. The Gonococcus fights back: is this time a knock out? Sex Transm Infect. 2010;86(6):415-21.

2. Tapsall JW, Ndowa F, Lewis DA, Unemo M. Meeting the public health challenge of multidrug- and extensively drugresistant Neisseria gonorrhoeae. Expert Rev Anti Infect Ther. 2009;7(7):821-34.
3. WHO Western Pacific Gonococccal Antimicrobial Surveillance Programme. Surveillance of antibiotic resistance in Neisseria gonorrhoeae in the WHO Western Pacific Region, 2006. Commun Dis Intell. 2008;32(1):48-51.

4. Kubanova A, Frigo N, Kubanov A, Sidorenko S, Lesnaya I, Polevshikova S, et al. The Russian gonococcal antimicrobial susceptibility programme (RU-GASP)--national resistance prevalence in 2007 and 2008, and trends during 2005-2008. Euro Surveill. 2010;15(14):pii=19533. Available from: http:// www.eurosurveillance.org/ViewArticle.aspx?Articleld =19533

5. Centers for Disease Control and Prevention. Sexually Transmitted Disease Surveillance 2009. Atlanta: U.S. Department of Health and Human Services; 2010. Available from: http://www.cdc.gov/std/statsog/toc.htm

6. Health Protection Agency. Gonococcal Resistance to Antimicrobials Surveillance Programme in England and Wales (GRASP): report of 2009 data. Health Protection Report. 2010;4(34). Available from: http://www.hpa.org.uk/hpr/ archives/2010/hpr3410.pdf

7. Cole MJ, Chisholm SA, Hoffmann S, Stary A, Lowndes CM, Ison CA, et al. European surveillance of antimicrobial resistance in Neisseria gonorrhoeae. Sex Transm Infect. 2010;86(6):427-32.

8. Australian Gonococcal Surveillance Programme. Annual Report of the Australian Gonococcal Surveillance Programme, 2009. Commun Dis Intell. 2010;34(2):89-95.

9. Bignell C, IUSTI/WHO. 2009 European (IUSTI/WHO) guideline on the diagnosis and treatment of gonorrhoea in adults. Int J STD AIDS. 2009;20(7):453-7.

10. Ito M, Yasuda M, Yokoi S, Ito S, Takahashi Y, Ishihara $\mathrm{S}$, et al. Remarkable increase in central Japan in 20012002 of Neisseria gonorrhoeae isolates with decreased susceptibility to penicillin, tetracycline, oral cephalosporins, and fluoroquinolones. Antimicrob Agents Chemother. 2004; $48(8): 3185-7$.

11. Tapsall JW. Neisseria gonorrhoeae and emerging resistance to extended spectrum cephalosporins. Curr Opin Infect Dis. 2009;22(1):87-91.

12. Barry PM, Klausner JD. The use of cephalosporins for gonorrhea: the impending problem of resistance. Expert Opin Pharmacother. 2009;10(4):555-77.

13. Golparian D, Hellmark B, Fredlund H, Unemo M. Emergence, spread and characteristics of Neisseria gonorrhoeae isolates with in vitro decreased susceptibility and resistance to extended-spectrum cephalosporins in Sweden. Sex Transm Infect. 2010;86(6):454-60.

14. Yokoi S, Deguchi T, Ozawa T, Yasuda M, Ito S, Kubota Y, et al. Threat to cefixime treatment for gonorrhea. Emerg Infect Dis. 2007;13(8):1275-7.

15. Unemo M, Golparian D, Syversen G, Vestrheim DF, Moi H. Two cases of verified clinical failures using internationally recommended first-line cefixime for gonorrhoea treatment, Norway, 2010. Euro Surveill. 2010;15(47):pii=19721. Available from: http://www.eurosurveillance.org/ViewArticle. aspx?Articleld $=19721$

16. Ison CA, Hussey J, Sankar KN, Evans J, Alexander S. Gonorrhoea treatment failures to cefixime and azithromycin in England, 2010. Euro Surveill. 2011;16(14): pii=19833. Available from: http://www.eurosurveillance.org/ViewArticle. aspx?Articleld $=19833$

17. St-Martin G, Cowan S, Hoffmann S. Gonorrhoea 2009. EPINews. 2010;36. [Accessed 13 September 2011]. Available from: http://www.ssi.dk/English/News/EPI-NEWS/2010/No\%20 36\%20-\%202010.aspx

18. Koedijk FD, van Veen MG, de Neeling AJ, Linde GB, van der Sande MA. Increasing trend in gonococcal resistance to ciprofloxacin in The Netherlands, 2006-8. Sex Transm Infect. 2010;86(1):41-5.

19. Tzelepi E, Avgerinou H, Flemetakis A, Siatravani E, Stathi $M$, Daniilidou $M$, et al. Changing figures of antimicrobial susceptibility and serovar distribution in Neisseria gonorrhoeae isolated in Greece. Sex Transm Dis. 2010;37(2):115-20.

20. Velicko I, Unemo M. Increase in reported gonorrhoea cases in Sweden, 2001 - 2008. Euro Surveill. 2009;14(34):pii=19315. Available from: http://www.eurosurveillance.org/ViewArticle. aspx?Articleld $=19315$

21. Eastick K. Gonococcal antibiotic surveillance in Scotland (GASS):prevalence, patterns and trends in 2009. HPS Weekly Report. 2010;44(34):339-42. Available from: http://www. documents.hps.scot.nhs.uk/ewr/pdf2010/1034.pdf

22. Martin IM, Hoffmann S, Ison CA. European Surveillance of Sexually Transmitted Infections (ESSTI): the first combined antimicrobial susceptibility data for Neisseria gonorrhoeae in Western Europe. J Antimicrob Chemother. 2006;58(3):587-93. 
23. European Centre for Disease Prevention and Control (ECDC). Sexually Transmitted Infections in 1990-2009. Stockholm:

ECDC; 2011. Available from: http://www.ecdc.europa. eu/en/publications/Publications/110526_SUR_STI_in_ Europe_1990-2009.pdf

24. European Centre for Disease Prevention and Control (ECDC). Gonococcal antimicrobial susceptibility surveillance in Europe 2009. Stockholm: ECDC; 2011. Available from: http:// ecdc.europa.eu/en/publications/Publications/1101_SUR_ Gonococcal_susceptibility_2009.pdf

25. Chisholm SA, Quaye N, Cole MJ, Fredlund H, Hoffmann S, Jensen JS, et al. An evaluation of gentamicin susceptibility of Neisseria gonorrhoeae isolates in Europe. J Antimicrob Chemother. 2011;66(3):592-5.

26. Unemo M, Fasth O, Fredlund H, Limnios A, Tapsall J. Phenotypic and genetic characterization of the 2008 WHO Neisseria gonorrhoeae reference strain panel intended for global quality assurance and quality control of gonococcal antimicrobial resistance surveillance for public health purposes. J Antimicrob Chemother. 2009;63(6):1142-51.

27. European Committee on Antimicrobial Susceptibility Testing (EUCAST). Breakpoint tables for interpretation of MICs and zone diameters. Version 1.3. EUCAST; 5 Jan 2011. [Accessed 13 September 2011]. Available from: http://www.eucast. org/fileadmin/src/media/PDFs/EUCAST_files/Disk_test documents/EUCAST_breakpoints_v1.3_pdf.pdf

28. Chisholm SA, Mouton JW, Lewis DA, Nichols T, Ison CA, Livermore DM. Cephalosporin MIC creep among gonococci: time for a pharmacodynamic rethink? J Antimicrob Chemother. 2010;65(10):2141-8

29. Tapsall J, Read P, Carmody C, Bourne C, Ray S, Limnios A, et al. Two cases of failed ceftriaxone treatment in pharyngeal gonorrhoea verified by molecular microbiological methods. J Med Microbiol. 2009;58(Pt 5):683-7.

30. Unemo M, Golparian D, Hestner A. Ceftriaxone treatment failure of pharyngeal gonorrhoea verified by international recommendations, Sweden, July 2010. Euro Surveill. 2011;16(6):pii=19792. Available from: http://www. eurosurveillance.org/ViewArticle.aspx?Articleld=19792

31. Bignell C, Garley J. Azithromycin in the treatment of infection with Neisseria gonorrhoeae. Sex Transm Infect. 2010;86(6):422-6. 